\section{Dynamique \\ et morphologie \\ mitochondriales}

\section{Acteurs, mécanismes et pertinence fonctionnelle}

Cécile Sauvanet, Laetitia Arnauné-Pelloquin,
Claudine David, Pascale Belenguer, Manuel Rojo
$>$ Les mitochondries sont des organites dynamiques qui se déplacent, se divisent et fusionnent continuellement. L'équilibre fusion-fission détermine si elles forment, dans la cellule, des filaments interconnectés ou apparaissent comme une collection de structures ponctiformes indépendantes. Les machineries de fusion et fission sont conservées des levures aux mammifères et comprennent trois GTPases de la famille des dynamines: Dnml/DRPl (nomenclature levure/ homme pour dynamin-related protein), impliquée dans la fission, et Fzol/MFN (mitofusine) et Mgml/OPAl (optic atrophy 1), requises pour la fusion. Alors que l'identification et la caractérisation des acteurs de la dynamique mitochondriale, de leur mécanisme d'action, de leurs fonctions et de leur régulation continuent à être l'objet de nombreuses recherches, la pertinence de ce processus est attestée par son rôle dans le fonctionnement mitochondrial, la survie cellulaire, le développement embryonnaire et son implication dans des maladies neurologiques. <

\section{Les mitochondries, des organites de morphologie longtemps méconnue}

Les mitochondries ont été identifiées il y a plus de cent ans par microscopie photonique, mais on a longtemps ignoré leur véritable morphologie et leur nature dynamique. Le nom qui leur a alors été attribué, dérivé du grec mitos (fil) et chondros (grain), reflétait l'hétérogénéité de leur morphologie. La microscopie électronique révéla ensuite qu'elles sont entourées de deux membranes (externe et interne) et possèdent un troisième système membranaire: les crêtes de la membrane interne [1]. Ces clichés de microscopie électronique, dévoilant des structures punctiformes qui ressemblaient, en taille et en morphologie, à leurs ancêtres les bactéries, conduisent une partie de la communauté scientifique à considérer les mitochon-

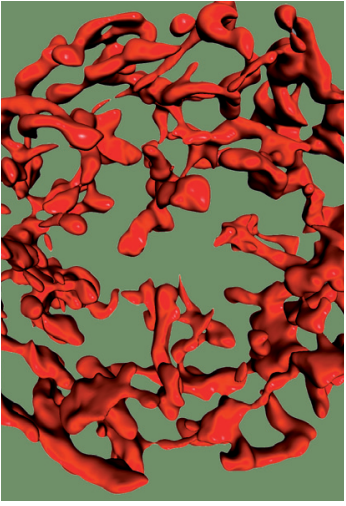

C. Sauvanet, C. David, M. Rojo : Institut de biochimie et génétique cellulaires (IBGC), UMR 5095 CNRS/

Université Victor Segalen, 1, rue Camille Saint-Saëns, 33077 Bordeaux Cedex, France.

L. Arnauné-Pelloquin,

P. Belenguer : Université de Toulouse, UPS, CNRS, UMR 52412,

Laboratoire métabolisme

dries comme de petites entités indépendantes. Ce concept pose des questions fondamenplasticité mitochondries, F-31000, France.

manuel.rojo@ibgc.cnrs.fr pascale.belenguer@cict.fr

tales en termes de génétique mitochondriale. Les génomes mitochondriaux coexistent-ils dans un seul compartiment ou sont-ils isolés dans des centaines de mitochondries indépendantes? Les mitochondries peuvent-elles réaliser des échanges moléculaires leur permettant de se complémenter? La redécouverte et la caractérisation de la morphologie et de la dynamique mitochondriales permettront de répondre à ces interrogations.

Le développement de nouvelles techniques de microscopie (microscopie électronique à tomographie, microscopie confocale) vers la fin du $x x^{e}$ siècle révéla à nouveau que les mitochondries peuvent adopter une structure punctiforme ou tubulaire. Dans les années 1990, des cribles génétiques menés chez la levure Saccharomyces cerevisiae ont conduit à l'isolement de mutants dont la morphologie des mitochondries était altérée et à l'identification des premières protéines impliquées dans la dynamique mitochondriale [2]. S. cerevisiae présente un réseau mitochondrial filamenteux qui résulte d'un équilibre dynamique entre deux forces antagonistes de fission et de fusion (Figure IA, $B)$. Dans des conditions normales, l'équilibre fusion/ 


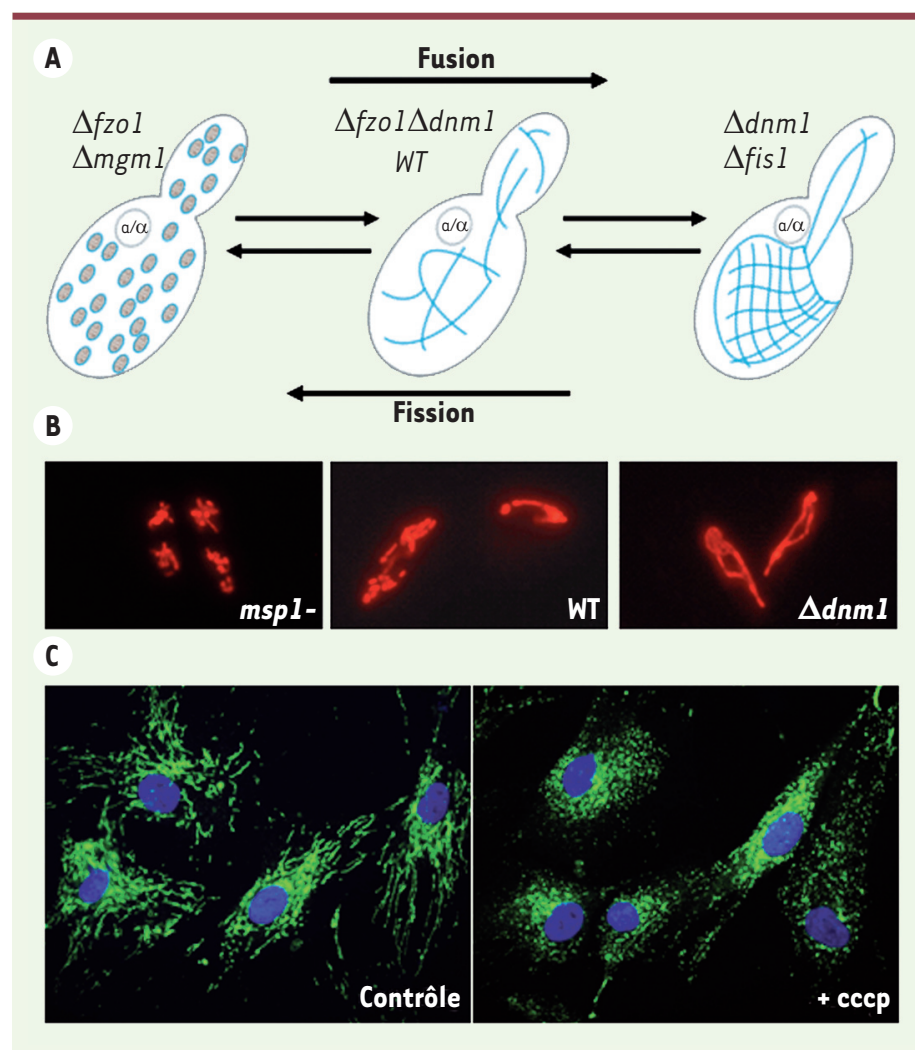

fission s'établit au profit de la fusion et les mitochondries apparaissent filamenteuses. L'inhibition de la composante fusion provoque la fragmentation des mitochondries. À l'inverse, lorsque la fission est inhibée, le réseau mitochondrial apparait excessivement filamenteux et interconnecté. De façon surprenante, un réseau mitochondrial de type normal est restauré quand à la fois la fusion et la fission sont altérées (Figure 1A). La plasticité de la morphologie des mitochondries est conservée dans les cellules eucaryotes supérieures dont les mitochondries apparaissent comme un réseau de filaments interconnectés quand la force de fusion prédomine, ou comme des structures punctiformes isolées quand la force de fission prévaut (Figure IC). En outre, l'observation des mitochondries par microscopie en temps réel montre clairement que cet équilibre n'est pas figé. Quelle que soit leur morphologie, les mitochondries se déplacent, se divisent et fusionnent continuellement $(\rightarrow)$.

\section{$(\rightarrow)$ Voir le film} disponible sur le site de Médecine/Sciences www.medecinesciences. org $^{1}$
Cet article récapitule nos connaissances sur la fusion et la fission mitochondriales, le composant dit intrinsèque de la dynamique mitochondriale. Une seconde composante, dite extrinsèque, contrôle la distribution et la mobilité des mitochondries via des interactions avec le cytosquelette [3]. Deux autres articles de ce numéro discutent la pertinence physiopathologique de la dynamique intrinsèque ainsi que son rôle dans l'apoptose $(\rightarrow)[40,41]$.

$\rightarrow$ Voir l'article de Céline

Castanier et Damien Arnoult, page 830 de ce numéro, et l'article de Guy Lenaers et al., page 836 de ce numéro

http://www.edk.fr

Figure 1. La morphologie mitochondriale, très variable, est gouvernée par l'équilibre fusion-fission. A, B. Les levures Saccharomyces cerevisiae (A) et Schizosaccharomyces pombe (B) sauvages présentent un réseau mitochondrial filamenteux. Lorsque la fusion est inhibée ( $\Delta \mathrm{fzol}, \Delta \mathrm{mgml}$, mspl-), les mitochondries sont fragmentées alors qu'elles forment de très longs filaments interconnectés lorsque la fission est inhibée $(\Delta \mathrm{dnml}, \Delta$ fisl). Une morphologie filamenteuse «normale» est restaurée lorsque l'on inhibe simultanément fusion et fission ( $\Delta \mathrm{fzol}, \Delta \mathrm{dnml}$ ). C. Dans des fibroblastes cutanés humains, les mitochondries, filamenteuses dans des conditions normales de culture (contrôle), se fragmentent lorsque la fusion est inhibée par dissipation du potentiel de membrane mitochondrial (+cccp).

\section{Les mitochondries fusionnent et fissionnent continuellement}

Les premiers tests de fusion mitochondriale, in vivo et in vitro, ont été réalisés chez $S$. cerevisiae $[2,4]$. Cette levure existe sous deux formes haploïdes capables de se conjuguer. Le marquage des mitochondries par des fluorophores différents a mis en évidence que les membranes mitochondriales des deux formes de levure fusionnent et permettent ainsi le mélange des composants mitochondriaux (Figure 2A). Ces études ont révélé que si tous les composants mitochondriaux sont échangés lors de la fusion, la mobilité des génomes mitochondriaux est réduite. La reconstitution de la fusion in vitro a démontré que la fusion des membranes internes et celle des membranes externes sont des événements distincts qui nécessitent tous deux un apport de GTP.

Nos connaissances sur la fusion mitochondriale chez les mammifères sont issues de travaux ex vivo. Des molécules fluorescentes sont adressées aux mitochondries de deux populations de cellules dont la fusion est induite. Après fusion cellulaire, l'analyse du mélange des molécules fluorescentes entre mitochondries met en évidence la fusion mitochondriale (Figure 2B, C). Ces études ont permis de démontrer que la fusion mitochondriale (1) permet l'échange de protéines, de complexes respiratoires et de nucléoïdes, (2) ne dépend pas du cytosquelette et (3) est abolie par une dissipation du potentiel de membrane mitochondrial $\left(\Delta \psi_{m}\right)[5,6]$. Elles ont également dévoilé que les membranes externe et interne fusionnent en deux réactions séparées et que seule la dernière est sensible à la dissipation du $\Delta \psi_{\mathrm{m}}$ [7]. Récemment, l'obtention de nouvelles protéines fluorescentes a permis d'étudier la fusion mitochondriale à l'intérieur d'une cellule. Des cellules exprimant une protéine fluorescente photoconvertible sont partiellement 


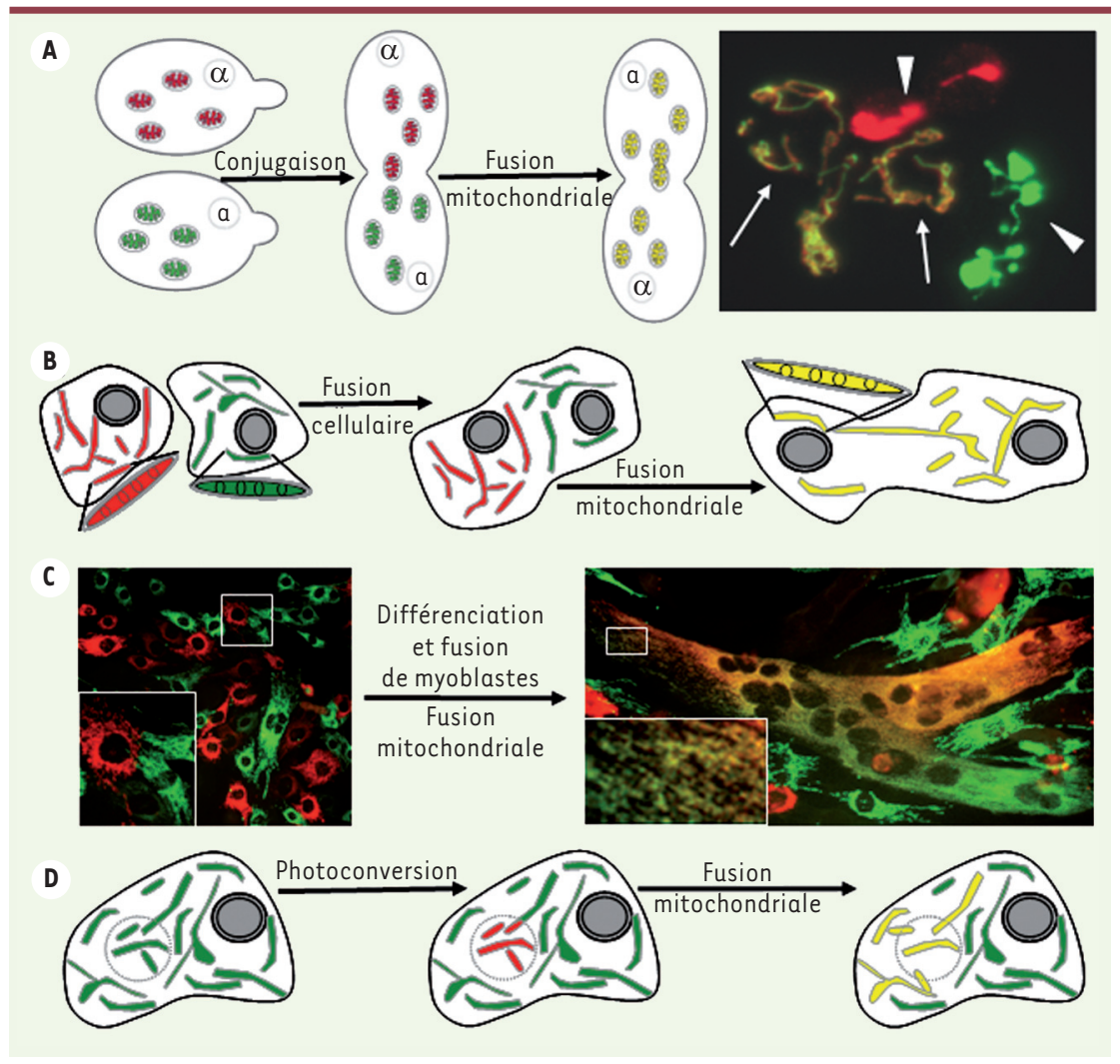

Figure 2. Tests utilisés pour l'étude de la fusion mitochondriale basés sur l'échange de protéines fluorescentes entre mitochondries. A. Dans la levure S. cerevisiae, la fusion est observée lors de la conjugaison de cellules haploïdes. Pointes de flèche : cellules haploïdes contenant des mitochondries simplement marquées. Flèches: zygote contenant des mitochondries doublement marquées. $\boldsymbol{B}$, C. Dans des cellules mammifères, la fusion cellulaire est induite par le polyéthylène glycol (B) ou a lieu lors de la différenciation de myoblastes en culture (C). D. L'utilisation de protéines photoconvertibles permet d'observer la fusion à l'intérieur d'une cellule.

irradiées par un laser, ce qui conduit au marquage de quelques mitochondries dont le devenir est suivi (Figure 2D). Ces expériences ont révélé l'existence de deux types de fusion [8]. Dans la majorité des cas, la fusion est instable (de type «kiss and run ») : les mitochondries fusionnent leurs deux membranes, échangent des composants et se séparent presque immédiatement. Dans une minorité des cas, la fusion est stable et conduit à la formation d'une mitochondrie allongée. Le processus de fission, pourtant clairement visible en microscopie en temps réel, n'a pas été caractérisé à ce jour à l'aide de tests dédiés. Dans la plupart des cas, la fission est observée indirectement par l'altération de l'équilibre fusion-fission et ses répercussions sur la morphologie mitochondriale $[6,7]$.

L'observation des phénomènes de fusion et fission a fait émerger de nombreuses questions. Quels sont les acteurs impliqués dans la fusion et la fission? Quels sont les mécanismes moléculaires sous-jacents? Quelle est la pertinence fonctionnelle de la dynamique mitochondriale? Comment est-elle régulée? Nous verrons que les réponses à toutes ces questions n'ont pas encore été obtenues.

\section{Les acteurs et la mécanistique}

Les protéines impliquées dans la fusion et la fission mitochondriales sont, dans la plupart des cas, spécifiques aux mitochondries et différentes dans leur structure et leur mécanisme d'action de celles qui sont impliquées dans le trafic vésiculaire entre organites. Bon nombre d'entre elles s'avèrent être conservées chez tous les eucaryotes (Figure 3A) [2].

La machinerie de fission, chez la levure, est composée de quatre protéines principales: Dnml, GTPase de la famille des dynamines, Fis l, Mdvl et Caf4 (Figure 3A, B). La délétion ou la mutation de Dnml, ou de ses homologues DRPI ou DLPI chez les métazoaires, augmente la longueur et la connectivité des mitochondries et empêche leur fragmentation $[4,9,10]$. Dnml, majoritairement cytosolique, est recrutée à la membrane mitochondriale externe au niveau des futurs sites de fission. Chez la levure, ce recrutement implique la protéine intégrale de la membrane externe Fisl et les protéines adaptatrices Mdvl et Caf4 [4] (Figure 3B). Le recrutement de DRP1 demeure assez obscur, d'une part car il n'existe pas d'orthologues mammifères de Caf 4 et Mdvl et d'autre part car la perte de l'homologue humain de Fisl ne perturbe pas la localisation mitochondriale de la dynamine [10]. Une fois recrutée à la membrane externe, Dnml/DRPl oligomérise et forme une spirale dont le changement de structure, induit par l'hydrolyse du GTP, provoque la fission des mitochondries (Figure 3B). La possibilité de découpler la fission des deux membranes mitochondriales chez C. elegans [11] suggère l'existence d'une machinerie indépendante pour la fission de la membrane interne. Cependant, l'existence d'une telle machinerie n'a pas encore été réellement démontrée. Curieusement, la machinerie de fission mitochondriale est également impliquée dans la fission des peroxysomes [12]. 
A

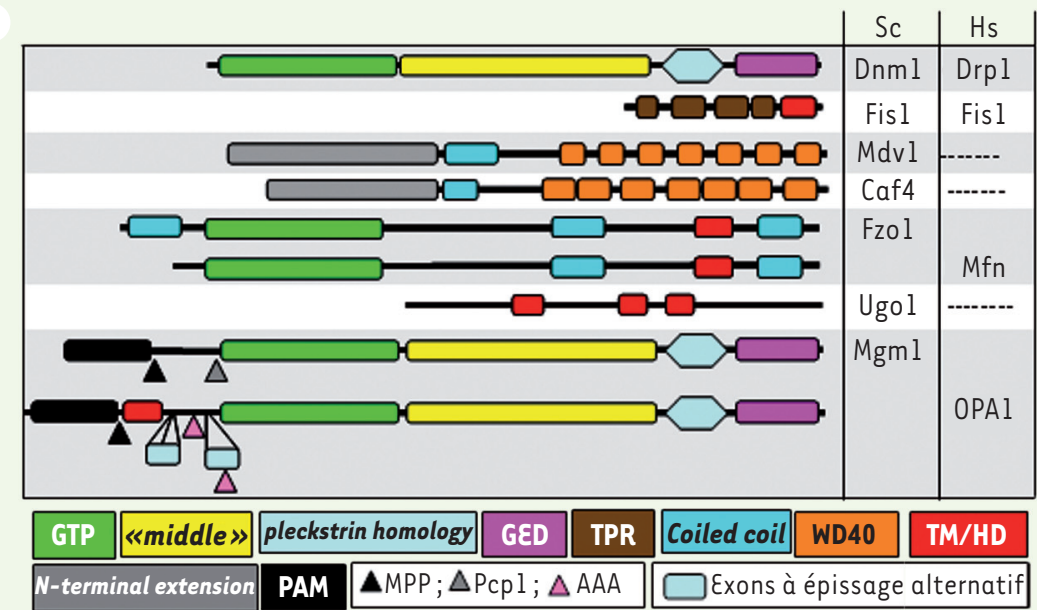

B
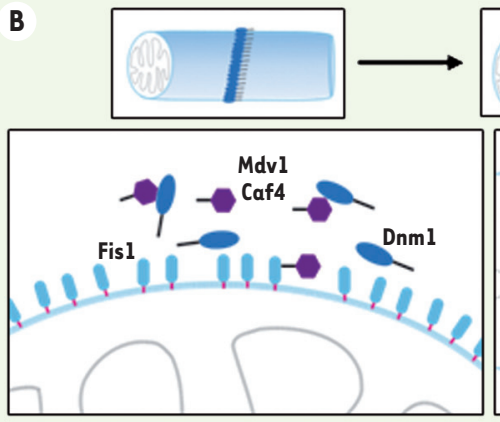

c
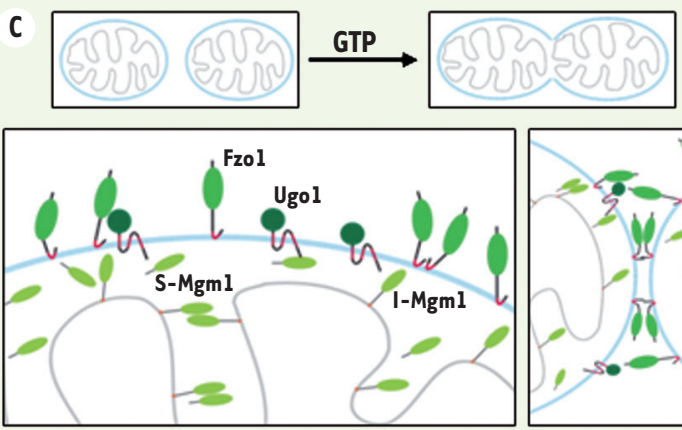
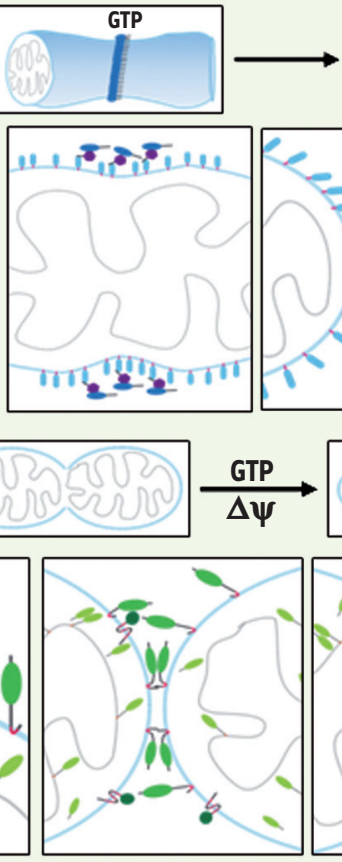
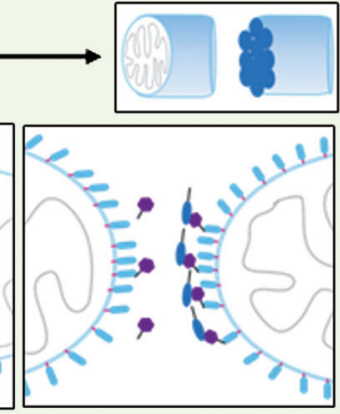

n.
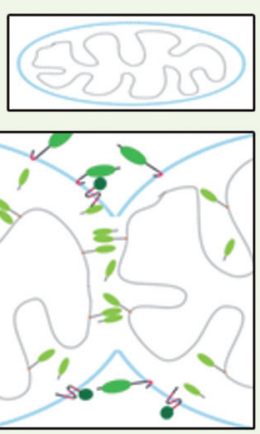

Figure 3. Acteurs et mécanistique de la fission et de la fusion. A. Structure des principales molécules impliquées dans la dynamique mitochondriale chez la levure (Sc) et l'homme (Hs). GTP: domaine de liaison et d'hydrolyse du GTP; «middle»: domaine «middle» des dynamines; GED: GTPase effector domain; TPR: tetratricopeptide repeat; TM/HD : domaine transmembranaire/hydrophobe; PAM: pré-séquence d'adressage mitochondrial; MPP/Pcpl/AAA: sites de clivage pour différentes protéases intra-mitochondriales : peptidase matricielle (MPP)/Pcpl/AAA protéase (AAA). B. Dnml est recrutée à la membrane interne au niveau des futurs sites de fission grâce à son interaction avec Mdvl et Caf4 qui interagissent elles-mêmes avec Fisl. L'oligomérisation de Dnml en spirale dont la structure est modifiée sous l'influence de l'hydrolyse du GTP va provoquer l'étranglement et la scission des mitochondries. C. Mécanistique de la fusion: la fusion des membranes externes dépend du GTP; celle des membranes internes du GTP et du $\mathrm{m} \Delta \psi$. Elles impliquent respectivement Fzol et Ugo, deux protéines intégrales de la membrane externe, et deux formes de Mgml, une longue (I-mgml) ancrée à la membrane interne, et une courte (s-mgml) en périphérie de la membrane interne ou libre dans l'espace intermembranaire. Fzol et Mgml contribuent à la fusion séquentielle de la membrane externe puis de la membrane interne par deux processus distincts impliquant d'abord une homodimérisation en trans de chacune des protéines, puis un mélange des bicouches lipidiques. Ugol, en interagissant avec Fzol et Mgml, couplerait la dynamique des deux membranes.
Le cœur de la machinerie de fusion mitochondriale de la levure est constitué par trois protéines: Fzol et Ugol, ancrées dans la membrane externe, et Mgml localisée au niveau de la membrane interne (Figure 3A, C). II est intéressant de remarquer que le premier homologue de Fzo fut découvert chez un mutant de la drosophile dont les mâles étaient stériles. Le nom Fzo vient du fait que les mitochondries des spermatozoïdes avaient l'apparence de tranches d'oignons désorganisées (fuzzy onions [13]). Les cellules de mammifères contiennent deux homologues de Fzol appelés mitofusines (MFN1 et MFN2 [14]), un homologue de Mgml appelé OPAl [15, 16], mais aucun homologue de Ugol. La délétion des gènes codant les protéines fusogènes entraîne, chez la levure et les mammifères, la fragmentation des mitochondries par inhibition de leur fusion [4]. On pense actuellement que les protéines Fzol/MFN s'associent en trans pour induire l'accolement puis la fusion, GTP-dépendante, des membranes externes, et que la fusion des membranes internes est ensuite prise en charge par la dynamine Mgml/OPAl (Figure 3C) [4]. Chez la levure, la dimérisation en trans de Mgml est nécessaire pour l'accolement et la fusion des deux membranes internes, mais chez les mammifères, la présence d'OPAl n'est requise que sur une seule des deux mitochondries [16]. Ugol, protéine intégrale de la membrane externe, interagit avec Fzol et Mgml et pourrait ainsi coupler la dynamique des deux membranes. Ugol influence à la fois la fusion de la membrane externe et celle de la membrane interne au cours d'une étape postérieure à l'accolement des membranes, probablement en facilitant le mélange des bicouches lipidiques [17]. L'absence d'un orthologue mammifère de 
Ugol pourrait expliquer pourquoi le couplage de la dynamique des deux membranes semble moins efficace chez les mammifères.

À côté des composants principaux des machineries de fusion et fission que nous venons de décrire, et qui sont globalement conservés de la levure à l'homme, d'autres acteurs de la dynamique mitochondriale, qui sont souvent spécifiques aux levures ou aux mammifères, ont également été identifiés [4]. Par exemple, chez les mammifères, la protéine de fission GDAPl (gangliosideinduced differentiation-associated protein 1 ), responsable d'une neuropathie chez l'homme $(\rightarrow$ ) et ATAD3A (ATPase family, AAA domain containing $3 A$ ), qui joue un rôle dans l'établissement et/ ou la régulation des interactions entre les membranes externe et interne [18]. Chez la levure, les protéines Mdm 10, Mdm 12 et Mmml, identifiées originellement comme des protéines de tubulation, viennent d'être impliquées dans l'interaction entre les mitochondries et le réticulum endoplasmique [19]. Ces protéines n'ont pas d'homologues chez les mammifères chez lesquels les interactions entre réticulum endoplasmique et mitochondries impliquent MFN2 [20].

\section{Régulation de la fusion/fission des mitochondries}

Des études pionnières de Hackenbrock ont révélé que l'ultrastructure des mitochondries est modulée par le métabolisme énergétique, et qu'elles peuvent adopter des conformations très différentes [21]. L'équilibre fusion/ fission et la morphologie des mitochondries sont également régulés par la bioénergétique mitochondriale et cellulaire [22], changent durant divers processus de différenciation [10] et sont altérés $(\rightarrow)$ Voir l'article de dans de nombreuses maladies $(\rightarrow)$. Guy Lenaers et al., page 836 de ce numéro

\begin{tabular}{|c|c|c|c|}
\hline Espèce & Protéine & Phénotype & Références \\
\hline $\mathrm{Sc}$ & Frol & Délétion: perte de l'ADNmt et de la capacité respiratoire & [2] \\
\hline $\mathrm{Ce}$ & fzo & KO : croissance ralentie. Létalité embryonnaire & [32] \\
\hline \multirow[t]{2}{*}{$\mathrm{Dm}$} & Fzo-spermatid & Mutation : développement des spermatozoïdes altéré, stérilité & [13] \\
\hline & Mfn-ubiquitaire & KD ubiquitaire : létal & [33] \\
\hline \multirow[t]{3}{*}{$\mathrm{Mm}$} & Mfnl, Mfn2 & KO ubiquitaire Mfnl ou Mfn2 : létalité embryonnaire & {$[34]$} \\
\hline & & KO Mfnl et Mfn2 en cellule : défauts de phosphorylation oxydative & {$[10]$} \\
\hline & & KO Mfn2 conditionnel : dégénérescence du cervelet & [34] \\
\hline $\mathrm{Sc}$ & Mgml & Délétion: perte de l'ADNmt et de la capacité respiratoire & [2] \\
\hline $\mathrm{Ce}$ & eat-3 & KO : croissance ralentie. Létalité embryonnaire & {$[32]$} \\
\hline \multirow[t]{2}{*}{$\mathrm{Dm}$} & OPAl & KO homozygote : létalité embryonnaire & [35] \\
\hline & & KO hétérozygote: anomalies dans la vision et dans de multiples organes & \\
\hline Hs & OPAl & KD en cellule: structure des crêtes altérée. Apoptose augmentée & [36] \\
\hline $\mathrm{Mm}$ & OPAl & Mutation : altération du nerf optique et de la vision & {$[37,38]$} \\
\hline Sc & Dnml & KO : stabilité de l'ADNmt diminuée & {$[39]$} \\
\hline $\mathrm{Ce}$ & Drpl & KO : croissance ralentie. Létalité embryonnaire & [32] \\
\hline $\mathrm{Dm}$ & Dnml & KD : majoritairement létal & {$[33]$} \\
\hline \multirow[t]{2}{*}{$\mathrm{Mm}$} & Drpl & KO : létalité embryonnaire & [9] \\
\hline & & $\begin{array}{l}\text { KO conditionnel : hypoplasie cérébrale, défaut de développement du cervelet et létalité } \\
\text { postnatale }\end{array}$ & \\
\hline
\end{tabular}

Tableau I. Conséquences de la mutation, délétion, knock-out (KO) ou knock-down (KD) des principaux gènes impliqués dans la fusion ou la fission mitochondriale. Espèces étudiées: Caenorhabditis elegans (Ce); Drosophila melanogaster (Dm); Homo sapiens (Hs); Mus musculus (Mm) et Saccharomyces cerevisiae (Sc); KD : knock-down; KO : knock-out; ADNmt: ADN mitochondrial ; Mfn : mitofusine ; 0PAl : optic atrophy 1 ; Dnml/ Drpl : dynamin-related protein 1. 
En outre, les mitochondries se fragmentent pendant l'apoptose $(\rightarrow)$ et s'allongent pendant la sénescence [10]. Malgré l'accumulation de données sur les acteurs et la mécanistique de la dynamique mitochondriale, nous ne savons que très peu de choses sur les signaux extra ou intracellulaires auxquels ce processus est soumis et sur les méca- $\rightarrow$ Voir l'article de Céline Castanier et Damien Arnoult, page 830 de ce numéro nismes par lesquels il est modulé. Récemment, l'existence de différentes voies qui altèrent le niveau d'expression (stabilité/dégradation), l'activité et/ou l'association membranaire des acteurs de fusion et de fission a été mise en évidence.

Chez les mammifères, une ubiquitine ligase mitochondriale (MITOL/MARCHV) interagit avec FISl et DRPl, qu'elle ubiquitinyle et dont elle régule les niveaux. DRPI semble être le substrat d'autres ubiquitine ligases et ses propriétés (activité, recrutement, stabilité) sont également modifiées par phosphorylation et SUMOylation [23]. Chez la levure, les niveaux de Fzol sont contrôlés par un processus de dégradation indépendant ou dépendant du protéasome [24, 25]. Chez les mammifères, MFN2 est phosphorylée par la protéine kinase A (PKA), interagit avec une ubiquitine ligase (MITOL/ MARCH5) et son niveau est augmenté par des inhibiteurs du protéasome [10]. L'activité, l'association membranaire et les fonctions de Mgml/OPAl sont modulées par l'existence de nombreuses isoformes de la dynamine [16]. Chez l'homme, un épissage alternatif du gène OPAl génère huit ARN messagers codant pour des isoformes protéiques impliquées soit dans la dynamique mitochondriale, soit dans l'apoptose [16]. Une protéolyse sélective et contrôlée de la région amino-terminale de Mgml/OPAl ou de leur orthologue Mspl chez S. pombe génère des isoformes longues et courtes, toutes deux nécessaires à la fonction de la dynamine [16, 26, 27]. Chez la levure, cette protéolyse est régulée par les niveaux d'ATP mitochondrial [26], chez les mammifères, elle dépend des niveaux d'ATP et est induite lors d'une dissipation du $\Delta \psi_{\mathrm{m}}$ ou en situation d'apoptose [28, 29].

\section{Pertinence fonctionnelle}

La pertinence fonctionnelle de la dynamique mitochondriale est illustrée par les nombreuses répercussions de son inactivation aussi bien au niveau de l'organite, de la cellule que de l'organisme (Tableau I). Ainsi, le fonctionnement de la chaîne respiratoire, l'homéostasie et la signalisation calcique, la production d'espèces réactives de l'oxygène ainsi que la différenciation musculaire, la plasticité et le fonctionnement des neurones, le processus de sénescence et la cascade apototique, sont altérés par des modifications de l'expression des différents acteurs de la dynamique $(\rightarrow)$ Voir l'article de Céline Castanier et Damien Arnoult, page 830 de ce numéro mitochondriale $(\rightarrow)$ [10].

Chez la levure, de nombreux mutants de fusion ou de tubulation présentent un défaut de maintien du génome mitochondrial [2], et c'est grâce à ce phénotype mutant que $\mathrm{Mgml}$ a été initialement découverte. Plus récemment, des altérations de la dynamique mitochondriale chez les mammifères ont été associées à des défauts dans l'organisation, le maintien et la transmission de l'ADNmt [30].

D'autre part, les avantages conférés spécifiquement par la fusion incluent les échanges moléculaires et la complémentation fonctionnelle entre mitochondries, la formation de filaments capables de transmettre de l'énergie sous forme de $\Delta \psi_{\mathrm{m}}$ à l'intérieur de la cellule [31]. À l'inverse, la fission des mitochondries favorise leur transport, notamment dans les neurones, leur répartition équitable au cours de la mitose et leur élimination par autophagie [30]. Enfin, la nature essentielle de la dynamique mitochondriale a définitivement été confirmée par l'implication de plusieurs acteurs de la dynamique mitochondriale dans des maladies neurodégénératives et par son absolue nécessité au cours du développement embryonnaire [30,31].

\section{Discussion et perspectives}

Depuis l'identification dans les années 1990 des protéines impliquées dans la dynamique mitochondriale, leur liste n'a cessé de croître. Très récemment sont venues s'ajouter Pink-1 (PTEN-induced kinase 1 ) et Parkine ( $\varepsilon 3$ ubiquitin ligase), deux protéines mutées dans des formes familiales de maladie de Parkinson. Ces protéines seraient à la fois impliquées dans la dynamique mitochondriale et dans la biogenèse/dégradation des mitochondries [30]. Nous pouvons espérer, dans les années à venir, que de nouveaux facteurs de fusion et fission seront découverts et que les protéines étudiées à ce jour, in vivo ou ex vivo, seront fonctionnellement caractérisées in vitro. La caractérisation d'un processus de fusion catalysé par des dynamines, protéines impliquées dans la fission, promet de révéler un nouveau mécanisme de fusion membranaire différent de ceux qui sont dépendants des SNARE (un acronyme dérivé de SNAP [soluble NSF attachment protein] receptors) intracellulaires ou des protéines de fusion virales.

Alors que les nombreuses conséquences de l'inactivation des acteurs qui contrôlent la dynamique mitochondriale illustrent l'importance physiologique de ce processus, il est à présent indispensable d'en clarifier les fondements moléculaires. Ceci devrait permettre de déterminer la part réelle de la dynamique mitochondriale dans ces différents processus et de déterminer, à l'image de OPAl et de MFN2, si deux fonctions distinctes sont portées par la même protéine (dynamique mitochondriale et apoptose dans le premier cas, et dynamique mitochondriale et métabolisme mitochondrial dans le deuxième cas).

La mise en évidence de mécanismes variés qui contrôlent l'expression des acteurs de la dynamique mitochondriale permet de supposer que ce processus est contrôlé par de multiples circuits. Par exemple, le recrutement et la stabilité de DRP1, régulés par sa phosphorylation par la PKA et sa déphosphorylation par la calcineurine [23], positionnent la fission mitochondriale au carrefour de voies de signalisation AMPc et $\mathrm{Ca}^{2+}$ dépendantes. Notre vue des mécanismes de régulation de la dynamique mitochondriale restant très parcellaire, gageons que de grandes avancées seront réalisées dans ce domaine. La connaissance des mécanismes de régulation de 
la dynamique mitochondriale permettra de mieux comprendre les nombreux processus qui en dépendent, ceci dans un contexte normal ou pathologique $(\rightarrow)$. $\diamond$

\section{$(\rightarrow)$ Voir l'article de Guy Lenaers et al., page 836 de ce numéro}

\section{SUMMARY}

Mitochondrial morphology and dynamics:

actors, mechanisms and functions

Mitochondria are dynamic organelles that continuously move, fuse and divide. Their overall morphology, ranging from a filamentous network to a collection of isolated dots, is determined by fusion-fission equilibrium, which depends on the cellular and physiological context. The machineries of fusion and fission, that are conserved throughout evolution, include three large GTPases of the dynamin-superfamily: Dnml/DRPl - involved in fission - as well as Fzol/MFN and Mgml/OPAl - required for fusion. While the activities, mecanisms and regulations of mitochondrial fusion and fission machineries continue to be unravelled, the relevance of mitochondrial dynamics is witnessed by their impact on organelle functions, cell survival and cell differenciation, their requirement for embryonic development and their involvement in neurological diseases. $\diamond$

\section{REMERCIEMENTS}

Manuel Rojo tient à remercier Anne Lombès (Hôpital de la Pitié-Salpêtrière, Paris) qui crut à ce projet quand la dynamique mitochondriale n'existait que sur papier. Nous remercions également les associations de patients pour leur soutien et leur confiance: Association française contre les myopathies (AFM), Rétina France, Association contre les maladies mitochondriales (AMMi), Ligue nationale contre le cancer comité Haute-Garonne, Association pour la Recherche sur le cancer (ARC).

\section{CONFLIT D'INTÉRÊTS}

Les auteurs déclarent n'avoir aucun conflit d'intérêts concernant les données publiées dans cet article.

\section{RÉFÉRENCES}

1. Frey TG, Mannella CA. The internal structure of mitochondria. Trends Biochem Sci $2000 ; 25: 319-24$.

2. Okamoto K, Shaw JM. Mitochondrial morphology and dynamics in yeast and multicellular eukaryotes. Annu Rev Genet 2005 ; 39 : 503-36.

3. Boldogh IR, Pon LA. Mitochondria on the move. Trends Cell Biol 2007 ; 17 : 502-10.

4. Hoppins S, Lackner L, Nunnari J. The machines that divide and fuse mitochondria. Annu Rev Biochem $2007 ; 76: 751-80$

5. Legros F, Malka F, Frachon P, et al. Organization and dynamics of human mitochondrial DNA. J Cell Sci $2004 ; 117: 2653-62$

6. Legros F, Lombes A, Frachon P, Rojo M. Mitochondrial fusion in human cells is efficient, requires the inner membrane potential and is mediated by mitofusins. Mol Biol Cell $2002 ; 13: 4343-54$.

7. Malka F, Guillery 0 , Cifuentes-Diaz C, et al. Separate fusion of outer and inner mitochondrial membranes. EMBO Rep $2005 ; 6$ : 853-9.

8. Liu X, Weaver D, Shirihai 0 , Hajnoczky G. Mitochondrial "kiss-and-run": interplay between mitochondrial motility and fusion-fission dynamics. EMBO J 2009 ; 28 : 3074-89.

9. Ishihara N, Nomura M, Jofuku A, et al. Mitochondrial fission factor Drpl is essential for embryonic development and synapse formation in mice. Nat Cell Biol 2009; 11 : 958-66.

10. Liesa M, Palacin M, Zorzano A. Mitochondrial dynamics in mammalian health and disease. Physiol Rev $2009 ; 89: 799-845$

11. Labrousse AM, Zappaterra MD, Rube DA, van der Bliek AM. C elegans dynamin-related protein DRP-1 controls severing of the mitochondrial outer membrane. Mol Cell 1999; 4 : 815-26.

12. Schrader M. Shared components of mitochondrial and peroxisomal division. Biochim Biophys Acta $2006 ; 1763: 531-41$.

13. Hales KG, Fuller MT. Developmentally regulated mitochondrial fusion mediated by a conserved, novel, predicted GTPase. Cell 1997; 90 : 121-9.

14. Rojo M, Legros F, Chateau D, Lombes A. Membrane topology and mitochondrial targeting of mitofusins, ubiquitous mammalian homologs of the transmembrane GTPase Fzo. J Cell Sci 2002 ; 115 : 1663-74.
15. Delettre C, Lenaers G, Griffoin JM, et al. Nuclear gene OPAl, encoding a mitochondrial dynamin-related protein, is mutated in dominant optic atrophy. Nat Genet $2000 ; 26: 207-10$.

16. Landes T, Leroy I, Bertholet A, et al. OPAl (dys)functions. Semin Cell Dev Biol $2010 ; 21: 593-8$.

17. Hoppins S, Horner J, Song C, et al. Mitochondrial outer and inner membrane fusion requires a modified carrier protein. J Cell Biol 2009; $184: 569-81$.

18. Gilquin B, Taillebourg $\varepsilon$, Cherradi N, et al. The AAA+ ATPase ATAD3A controls mitochondrial dynamics at the interface of the inner and outer membranes. Mol Cell Biol $2010 ; 30$ : 1984-96.

19. Kornmann B. Le complexe ERMES : une connexion haut débit entre le réticulum endoplasmique et les mitochondries. Med Sci (Paris) $2010 ; 26: 145-6$.

20. De Brito OM, Scorrano L. Mitofusin 2 tethers endoplasmic reticulum to mitochondria. Nature $2008 ; 456: 605-10$.

21. Hackenbrock CR, Rehn TG, Weinbach $\varepsilon C$, Lemasters JJ. Oxidative phosphorylation and ultrastructural transformation in mitochondria in the intact ascites tumor cell. J Cell Biol 1971; 51 : 123-37.

22. Sauvanet C, Duvezin-Caubet S, di Rago JP, Rojo M. Energetic requirements and bioenergetic modulation of mitochondrial morphology and dynamics. Semin Cell Dev Biol $2009 ; 21: 558-65$.

23. Santel A, Frank S. Shaping mitochondria: The complex posttranslational regulation of the mitochondrial fission protein DRP1. IUBMB Life 2008; 60 : 448-55.

24. Escobar-Henriques M, Langer T. Mitochondrial shaping cuts. Biochim Biophys Acta $2006 ; 1763: 422-9$.

25. Cohen MM, Leboucher GP, Livnat-Levanon N, et al. Ubiquitin-proteasomedependent degradation of a mitofusin, a critical regulator of mitochondrial fusion. Mol Biol Cell 2008; 19 : 2457-64.

26. Zick M, Duvezin-Caubet $S$, Schafer A, et al. Distinct roles of the two isoforms of the dynamin-like GTPase Mgml in mitochondrial fusion. FEBS Lett 2009; $583: 2237-43$.

27. Diot A, Guillou E, Daloyau M, et al. Transmembrane segments of the dynamin Msplp uncouple its functions in the control of mitochondrial morphology and genome maintenance. J Cell Sci 2009; $122: 2632-9$

28. Baricault L, Segui B, Guegand L, et al. OPAl cleavage depends on decreased mitochondrial ATP level and bivalent metals. Exp Cell Res 2007 ; 313 : 3800-8.

29. Guillery 0 , Malka F, Landes T, et al. Metalloprotease-mediated OPAl processing is modulated by the mitochondrial membrane potential. Biol Cell 2008; $100: 315-25$.

30. Chen H, Chan DC. Mitochondrial dynamics--fusion, fission, movement, and mitophagy--in neurodegenerative diseases. Hum Mol Genet 2009; 18 : R169-76.

31. Chan DC. Mitochondria: dynamic organelles in disease, aging, and development. Cell 2006; 125: 1241-52.

32. Breckenridge DG, Kang BH, Kokel D, et al. Caenorhabditis elegans drp-l and fis-2 regulate distinct cell-death execution pathways downstream of ced-3 and independent of ced-9. Mol Cell $2008 ; 31: 586-97$.

33. Deng H, Dodson MW, Huang H, Guo M. The Parkinson's disease genes pinkl and parkin promote mitochondrial fission and/or inhibit fusion in Drosophila. Proc Natl Acad Sci USA 2008; 105 : 14503-8.

34. Chen H, McCaffery JM, Chan DC. Mitochondrial fusion protects against neurodegeneration in the cerebellum Cell $2007 ; 130: 548-62$.

35. Tang S, Le PK, Tse S, et al. Heterozygous mutation of Opal in Drosophila shortens lifespan mediated through increased reactive oxygen species production. PLoS One $2009 ; 4$ : e4492.

36. Olichon A, Baricault L, Gas N, et al. Loss of OPAl perturbates the mitochondrial inner membrane structure and integrity, leading to cytochrome $c$ release and apoptosis. J Biol Chem 2003; 278 : 7743-6.

37. Davies VJ, Hollins AJ, Piechota MJ, et al. Opal deficiency in a mouse model of autosomal dominant optic atrophy impairs mitochondrial morphology, optic nerve structure and visual function. Hum Mol Genet $2007 ; 16$ : 1307-18.

38. Alavi MV, Bette S, Schimpf S, et al. A splice site mutation in the murine Opal gene features pathology of autosomal dominant optic atrophy. Brain 2007 ; $130: 1029-42$.

39. Hanekamp T, Thorsness MK, Rebbapragada I, et al. Maintenance of mitochondrial morphology is linked to maintenance of the mitochondrial genome in Saccharomyces cerevisiae. Genetics 2002 ; 162 : 1147-56.

40. Castanier C, Arnoult D. La dynamique mitochondriale au cours de l'apoptose. Med Sci (Paris) $2010 ; 26: 830-5$.

41. Lenaers $G$, Amati-Bonneau P, Delettre C. De la levure aux maladies neurodégénératives : dix ans d'exploration des pathologies de la dynamique mitochondriale. Med Sci (Paris) 2010 ; 26 : 836-41.

\section{TIRÉS À PART \\ M. Rojo}

